\title{
Alcohol drinking and risk of subsequent hospitalisation with pneumonia
}

\author{
J.B. Kornum*, K.M. Due\#, M. Nørgaard*, A. Tjønneland , K. Overvad ${ }^{\#,+}$, \\ H.T. Sørensen* and R.W. Thomsen*
}

ABSTRACT: The dose-response relationship between alcohol consumption and pneumonia risk in healthy individuals is poorly understood.

We examined 22,485 males and 24,682 females from Denmark who were aged 50-64 yrs. Subjects were without major chronic diseases at baseline and had a median follow-up of 12 yrs for first-time hospitalisation with pneumonia.

1,091 (males) and 944 (females) had pneumonia-related hospitalisation. Among males, the risk of pneumonia was increased for alcohol abstainers and those who drank large weekly amounts. The adjusted hazard ratios (HRs) for 0, 7-20, 21-34, 35-50 and >50 drinks $\cdot$ week $^{-1}$ were $^{1.49}$ (95\% Cl 1.00-2.21), 0.88 (95\% Cl 0.76-1.03), 0.87 (95\% Cl 0.72-1.05), 1.15 (95\% Cl 0.93-1.44) and 1.81 (95\% Cl 1.40-2.33), respectively, compared with 1-6 drinks week $^{-1}$. The association between high alcohol intake and pneumonia persisted after controlling for subsequent chronic diseases.

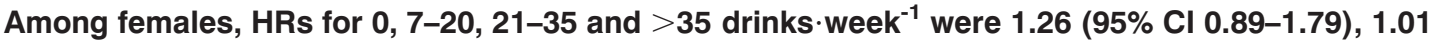
(95\% Cl 0.88-1.17), 1.10 (95\% Cl 0.88-1.37) and 0.54 (95\% Cl 0.29-1.01), respectively. For the same moderate to high weekly alcohol amount, infrequent intake yielded higher pneumonia HRs than more regular intake in both sexes.

Regular moderate alcohol intake is not associated with increased risk of hospitalisation for pneumonia. High weekly alcohol consumption in males and infrequent heavy drinking in both sexes may increase pneumonia risk.

KEYWORDS: Alcohol drinking, chronic diseases, epidemiologic study, pneumonia, risk

$\mathbf{P}$ neumonia is a major clinical and public health problem and a leading cause of death worldwide. Over the past two decades, hospitalisations with pneumonia have increased by $20-50 \%$ in ageing European and US populations [1-5].

Alcohol abuse has been associated with a two- to nine-fold higher risk of pneumonia [6-10], and alcohol consumption is high in Western populations [11]. Abuse of alcohol may increase susceptibility to pneumonia for several reasons. For example, alcohol intake may cause alterations in neutrophil and macrophage function and abnormalities in ciliary and surfactant functioning in the lung [12]. Alcohol overuse also can increase the risk of aspiration and suppress the normal cough reflex [12]. Finally, chronic alcohol intake is closely associated with malnutrition [12] and other chronic diseases that may affect pneumonia risk [13].

However, data are sparse on how the amount and frequency of alcohol intake are associated with increased pneumonia risk in apparently healthy individuals [10, 14-16]. A recent meta-analysis [10] based on only three studies [14-16] found that individuals who consumed alcohol in amounts corresponding to 14,35 and 70 drinks per week had relative risks for pneumonia of 1.1 (95\% CI 1.0 1.2), 1.3 (95\% CI 1.1-1.7) and 1.8 (95\% CI 1.1-2.8), respectively, compared with nondrinkers. Inadequate adjustment for smoking and other potential confounders may have affected these findings, and the studies also did not address whether the relationship resulted from alcohol consumption per se or from acquired chronic diseases. Moreover, frequency of alcohol consumption (i.e. regular versus binge drinking) has been shown to affect health differently $[17,18]$, but the effect of drinking frequency on pneumonia risk remains unknown. Finally, important sex differences in alcohol pharmacokinetics have been reported [19], and few studies on alcohol intake and pneumonia have examined this interaction $[15,16]$.

In-depth studies of these issues are needed to improve our understanding of the relationship between alcohol consumption and pneumonia risk. In this prospective cohort study, we examined the association between amount and frequency
AFFILIATIONS

*Dept of Clinical Epidemiology,

Clinical Institute,

${ }^{+}$Dept of Epidemiology, School of Public Health, Aarhus University,

"Dept of Cardiology, Center for

Cardiovascular Research, Aalborg Hospital, Aarhus University Hospital, Aarhus, and

"Danish Cancer Society,

Copenhagen, Denmark.

CORRESPONDENCE

J.B. Kornum

Dept of Clinical Epidemiology

Aarhus University Hospital

Sdr. Skowvej 15

DK-9000 Aalborg

Denmark

E-mail: j.kornum@rn.dk

Received:

Jan 032011

Accepted after revision:

May 182011

First published online:

June 092011

European Respiratory Journal

Print ISSN 0903-1936

Online ISSN 1399-3003 
of alcohol intake and risk of subsequent pneumonia-related hospitalisation among apparently healthy middle-aged males and females who were free from major chronic diseases at baseline.

\section{MATERIALS AND METHODS}

\section{Study population}

From December 1993 to May 1997, a total of 160,725 people (80,996 males and 79,729 females) were invited to participate in the Danish study entitled Diet, Cancer and Health. The study has been described in detail elsewhere [20, 21]. Eligible participants were aged 50-64 yrs, were born in Denmark, were residents of Copenhagen or Aarhus (both Denmark) and did not have a record of cancer in the Danish Cancer Registry. Potential participants were identified from the computerised records of the Civil Registration System in Denmark, which has records dating from 1968 on all Danish residents. Information maintained by the Civil Registration System includes name, address, vital status and a unique personal identification number encoding sex and date of birth. A total of 57,053 people (27,178 males and 29,875 females) agreed to participate in the Danish Diet, Cancer and Health Study. All participants completed a detailed food frequency questionnaire $[22,23]$ and a second baseline questionnaire concerning lifestyle factors.

For this study, we excluded 840 people who had experienced a hospitalisation for pneumonia before enrolment, 6,851 people who had other major chronic diseases diagnosed before enrolment (see below), 366 people with a missing baseline questionnaire or missing variables, and 1,829 people with inconsistent information (i.e. who reported no alcohol intake at all together with a reported alcohol drinking frequency greater than zero, or vice versa). Thus, data for a total of 47,167 people (22,485 males and 24,682 females) were included in our analysis. The study was approved by regional ethics committees in Copenhagen and Aarhus and by the Danish Data Protection Agency.

\section{Data on alcohol intake and drinking patterns}

In the lifestyle questionnaire, participants reported their usual frequency of alcohol intake using one of seven possible response categories: "never", "less than once per month", "one to three times per month", "once a week", "two to four times per week", "five to six times per week", and "daily" [18].

In the food frequency questionnaire, participants were asked to state their average quantity of alcohol consumed during the past year, in terms of intake of specific amounts of each beverage: light, normal and strong beer (number of bottles); red, white and fortified wine (number of glasses); and spirits (number of drinks). The possible response categories (for bottles of beer, glasses of wine or drinks of spirits) were: "no alcohol intake", "less than one per month", "one per month", "two to three per month", "one per week", "two to four per week", "five to six per week", "one per day", "two to three per day", "four to five per day", "six to seven per day", and "eight or more per day". Based on the ethanol content of the different beverage types, these exposure categories were converted into number of standard drinks (12 $\mathrm{g}$ alcohol) per week and then added to yield an average measure of total weekly alcohol intake [18]. We defined abstainers as participants who reported never drinking in both questionnaires.
For a supplementary analysis, we obtained information from the lifestyle questionnaires on abstainers' previous alcohol intake.

\section{Identification of first-time hospitalisation with pneumonia}

Study participants who had a first-time pneumonia-related hospitalisation were identified by linking their civil registration number to the Danish National Registry of Patients. This registry has collected data on all hospitalisations in Denmark since 1977; its records include the civil registration number and up to 20 discharge diagnoses. Physicians coded diagnoses according to the International Classification of Diseases 8th revision (ICD-8) until the end of 1993, and 10th revision (ICD10) thereafter. We identified all in-patients with the following diagnoses recorded between the date of enrolment into the cohort and April 10, 2008: pneumonia (J12.x-J18.x), ornithosis (A709.x) or legionellosis (A481.x) [3].

\section{Data on major chronic diseases prior to enrolment}

From the Danish National Registry of Patients, we obtained information on participants' major chronic diseases, defined as those included in the Charlson Comorbidity Index [24] (myocardial infarction, congestive heart failure, peripheral vascular disease, cerebrovascular disease, dementia, chronic pulmonary disease (including asthma), connective tissue disease, ulcer disease, liver disease, diabetes, hemiplegia, renal disease, cancer and AIDS), as well as previous diagnoses of HIV infection, gastro-oesophageal reflux, hypertension and alcoholism-related diseases. We categorised these diseases as diseases directly related to alcoholism, cancer, cardiovascular diseases, chronic pulmonary diseases and other diseases (see the Appendix for the ICD-8 and ICD-10 codes).

\section{Other potential confounders}

At baseline, trained laboratory technicians recorded anthropometric measurements. Body mass index (BMI) was calculated as weight $(\mathrm{kg})$ divided by height $\left(\mathrm{m}^{2}\right)$. Participants provided information on smoking, length of basic schooling and subsequent education in the baseline questionnaire.

\section{Statistical analysis}

We a priori categorised males and females according to total weekly alcohol intake (males: 0, 1-6, 7-20, 21-34, 35-50 and $>50$ drinks. week $^{-1}$; females: $0,1-6,7-20,21-35$ and $>35$ drinks. week $^{-1}$ ) and according to their weekly frequency of drinking alcohol (never, $\leqslant 1$ day, 2-4 days, 5-6 days, and 7 days a week) [17]. To ensure adjustment for confounding by age, we used age as the underlying time variable, with follow-up starting at participant age at recruitment. Follow-up extended until age on April 10, 2008, or until age at pneumonia diagnosis, death, or migration, whichever came first.

We computed pneumonia incidence rates by dividing the number of events by the accumulated person-time of follow-up within the groups defined by alcohol intake, separately for males and females. Cox's regression was used to compute hazard ratios (HRs) as measures of relative risk for hospitalisation with pneumonia according to alcohol intake level. Participants who consumed 1-6 drinks $\cdot$ week $^{-1}$ served as the reference group. We also controlled for baseline BMI $(<22.5,22.5-24.9$, 25.0-29.9, $30.0-34.9$ and $>35 \mathrm{~kg} \cdot \mathrm{m}^{-2}$ ) and smoking status (never, former

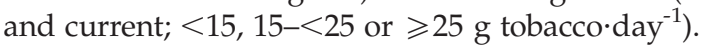


In a supplementary analysis, we adjusted for length of basic schooling (7, 8-10 and >10 yrs) and higher education (no higher education or short-, mid- or long-term higher education). Next, we examined the impact of chronic diseases diagnosed during follow-up, by including categories of diseases diagnosed during follow-up and before first pneumonia-related hospitalisation as time-dependent binary variables in the regression model. All analyses were repeated using frequency of alcohol consumption as the exposure (with those who consumed alcohol no more than one day per week serving as the reference). Finally, we computed HRs of pneumonia-related hospitalisation for different combinations of amount of alcohol consumption and drinking frequency. Data were analysed using Stata Statistical Software (Release 11 SE; Stata Corporation, College Station, TX, USA).

\section{RESULTS}

\section{Descriptive data}

A total of 1,091 males and 944 females had their first pneumoniarelated hospitalisation during median follow-up periods of 11.8 and 11.9 yrs, respectively. The corresponding incidence rates of hospitalisation with pneumonia were 4.25 and 3.28 per 1,000 person-yrs for males and females, respectively. Table 1 summarises participant baseline characteristics. At enrolment, median alcohol intake was 12 drinks $\cdot$ week $^{-1}$ (5th to 95th percentiles: 1-47 drinks $\cdot$ week $^{-1}$ ) among males and 6 drinks $\cdot$ week $^{-1}$ (5th to 95th percentiles: 1-25 drinks $\cdot$ week $^{-1}$ ) among females. In total, $338(1.5 \%)$ males and $644(2.6 \%)$ females were alcohol abstainers. Among male abstainers, $152(45.0 \%)$ reported having had a previous alcohol intake of $>21 \mathrm{drinks} \cdot$ week $^{-1}$ at some time

\begin{tabular}{|c|c|c|c|}
\hline \multirow[t]{2}{*}{ TABLE 1} & \multicolumn{3}{|c|}{$\begin{array}{l}\text { Baseline characteristics among females and } \\
\text { males in the Danish cohort followed for } \\
\text { pneumonia hospitalisations }\end{array}$} \\
\hline & & Females & Males \\
\hline \multicolumn{2}{|c|}{ Participants n } & 24682 & 22485 \\
\hline \multicolumn{2}{|l|}{ Age yrs } & $56(51-64)$ & $56(51-64)$ \\
\hline \multicolumn{2}{|c|}{ Alcohol intake drinks $\cdot$ week $^{-1}$} & $6(1-25)$ & $12(1-47)$ \\
\hline \multicolumn{2}{|c|}{ Body mass index $\mathrm{kg} \cdot \mathrm{m}^{-2}$} & $24.7(20.0-33.2)$ & $26.1(21.5-32.7)$ \\
\hline \multicolumn{4}{|c|}{ Education \% } \\
\hline \multicolumn{4}{|c|}{ Basic schooling } \\
\hline \multicolumn{2}{|l|}{$\leqslant 7 \mathrm{yrs}$} & 29.3 & 33.0 \\
\hline \multicolumn{2}{|l|}{$8-10$ yrs } & 51.1 & 42.1 \\
\hline \multicolumn{2}{|l|}{$>10 \mathrm{yrs}$} & 19.6 & 24.9 \\
\hline \multicolumn{4}{|c|}{ After basic schooling } \\
\hline \multicolumn{2}{|c|}{ No education } & 17.7 & 9.1 \\
\hline \multicolumn{2}{|c|}{ Short term } & 31.6 & 13.7 \\
\hline \multicolumn{2}{|c|}{ Mid term } & 38.9 & 42.1 \\
\hline \multicolumn{2}{|c|}{ Long term } & 11.9 & 35.2 \\
\hline \multicolumn{4}{|c|}{ Smoking status \% } \\
\hline \multicolumn{2}{|c|}{ Never smoked } & 45.1 & 27.0 \\
\hline \multicolumn{2}{|c|}{ Formerly smoked } & 23.4 & 34.4 \\
\hline \multicolumn{4}{|c|}{ Current smoker } \\
\hline \multicolumn{2}{|c|}{ 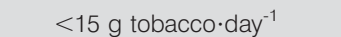 } & 14.9 & 10.5 \\
\hline \multicolumn{2}{|c|}{$15-<25 \mathrm{~g}$ tobacco $\cdot$ day $^{-1}$} & 14.1 & 17.0 \\
\hline \multicolumn{2}{|c|}{$\geqslant 25 \mathrm{~g}$ tobacco $\cdot$ day $^{-1}$} & 2.4 & 11.2 \\
\hline
\end{tabular}

Data are presented as median (5th-95th percentiles), unless otherwise stated. prior to enrolment in the Danish Diet, Cancer and Health Study, whereas 93 (14.4\%) of the female abstainers reported having had a previous alcohol intake of $>14$ drinks $\cdot$ week $^{-1}$.

\section{Risk of hospitalisation with pneumonia among males and females according to alcohol intake}

Among males, no alcohol intake at all and an intake $>50$ drinks. week $^{-1}$ were associated with a higher risk of pneumonia-related hospitalisation compared to the reference consumption of 1-6 drinks $\cdot$ week $^{-1}$ (tables 2 and 3). The corresponding adjusted HRs were 1.49 (95\% CI 1.00-2.21, $\mathrm{p}=0.05)$ for abstainers and 1.81 (95\% CI 1.40-2.33, p<0.001) for those consuming $>50$ drinks. week $^{-1}$, after adjustment for smoking status and BMI. Moderate alcohol consumption was associated with adjusted pneumonia HRs $<1$. Further adjustment for length of basic schooling and higher education yielded virtually identical HRs (data not shown). When chronic diseases diagnosed during follow-up were added to the model, the adjusted HR for pneumonia associated with an intake of $>50$ drinks week $^{-1}$ was 1.45 (1.11$1.90, p=0.01)$. Among females, alcohol abstinence was associated with an adjusted pneumonia HR of 1.26 (95\% CI 0.89-1.79, $\mathrm{p}=0.19$ ), whereas high consumption of $>35$ drinks $^{*}$ week $^{-1}$ was associated with an adjusted HR of 0.54 (95\% CI 0.29-1.01, $\mathrm{p}=0.06)$.

\section{Risk of hospitalisation with pneumonia according to weekly frequency of alcohol consumption}

Among males, drinking alcohol 2-6 days $\cdot$ week $^{-1}$ was associated with pneumonia hospitalisation HRs $<1$, compared with those drinking no more than 1 day week $^{-1}$ (table 4$)$. The adjusted HRs were $0.82(95 \%$ CI $0.69-0.97, \mathrm{p}=0.02)$ for drinking $2-4$ days.

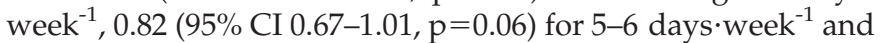
$1.05(95 \%$ CI $0.88-1.24, p=0.59)$ for 7 days $\cdot$ week $^{-1}$. Similar to males, females who consumed alcohol more often had a lower pneumonia risk than females who drank no more than 1 day week $^{-1}$ (table 4).

\section{Risk of hospitalisation with pneumonia according to combinations of drinking frequency and alcohol intake}

Table 5 lists the HRs of hospitalisation with pneumonia for different combinations of alcohol amount and drinking frequency. Within each category of drinking frequency, males who drank the largest amounts generally had the highest risk. Among males consuming $>20$ drinks $\cdot$ week $^{-1}$, HRs were generally higher for infrequent intake than for frequent intake within similar categories of amount. Similar results were observed for females (table 5).

\section{DISCUSSION}

In this large cohort study conducted among middle-aged Danes with no hospital-diagnosed chronic diseases at enrolment, we found that both total alcohol abstinence and high alcohol consumption were associated with a higher risk of pneumonia-related hospitalisation among males, but not among females. The increased risk conferred by heavy alcohol use in males was not exclusively explained by development of other chronic diseases during follow-up. Drinking frequency influenced the relationship between alcohol intake and pneumonia risk, i.e. for the same moderate to high average amount of alcohol intake, infrequent intake was associated with higher pneumonia risk than was frequent intake. 
TABLE 2 Hospitalisation rates for pneumonia among males according to alcohol intake, Denmark 1993-2008

\begin{tabular}{|c|c|c|c|c|c|c|}
\hline & \multicolumn{6}{|c|}{ Drinks $\cdot$ week $^{-1}$} \\
\hline & 0 & $1-6$ & $7-20$ & $21-34$ & $35-50$ & $>50$ \\
\hline Males n & 338 & 5346 & 9637 & 4469 & 1894 & 801 \\
\hline Pneumonia diagnosis $n$ & 26 & 272 & 406 & 193 & 114 & 80 \\
\hline $\begin{array}{l}\text { Incidence rate per } \\
1000 \text { person-yrs }\end{array}$ & 7.02 & 4.45 & 3.67 & 3.79 & 5.34 & 9.16 \\
\hline Crude HR $(95 \% \mathrm{Cl})^{\#}$ & $p=0.02$ & & $p=0.05$ & $p=0.22$ & $p=0.02$ & $\mathrm{p}<0.001$ \\
\hline \multirow[t]{2}{*}{ Adjusted HR $(95 \% \mathrm{Cl})^{\top}$} & $1.49(1.00-2.21)$ & 1.0 (ref.) & $0.88(0.76-1.03)$ & $0.87(0.72-1.05)$ & $1.15(0.93-1.44)$ & $1.81(1.40-2.33)$ \\
\hline & $p=0.05$ & & $p=0.11$ & $p=0.14$ & $p=0.20$ & $\mathrm{p}<0.001$ \\
\hline \multirow[t]{2}{*}{ Adjusted HR $(95 \% \mathrm{Cl})^{+}$} & $1.40(0.93-2.12)$ & 1.0 (ref.) & $0.94(0.80-1.10)$ & $0.90(0.75-1.09)$ & $1.14(0.91-1.43)$ & $1.45(1.11-1.90)$ \\
\hline & $p=0.11$ & & $p=0.42$ & $p=0.30$ & $p=0.24$ & $p=0.01$ \\
\hline
\end{tabular}

HR: hazard ratio; Cl: confidence interval; ref.: reference. \#: calculated using Cox's regression, with age as the underlying time variable; ": based on the crude model with additional adjustment for smoking status and body mass index; ${ }^{+}$, based on the crude model with additional adjustment for smoking status, body mass index and major chronic diseases.

The strengths of our study include its large size, the ability to adjust for related lifestyle factors such as smoking and BMI, and the virtually complete follow-up for pneumonia-related hospitalisations. Regarding weaknesses, similar to other cohort studies on this topic, we based our information on self-reported alcohol use at the time of enrolment, and we cannot exclude misclassification stemming from inaccurate reporting. Underreporting of alcohol intake or drinking frequency could have led to overestimation of the relative risks. Alcohol consumption was estimated based on the type of drink and, because drinks can vary in their alcohol content, some misclassification may have occurred. Moreover, drinking habits among study participants may have changed over time.

Another potential limitation is the study's reliance on discharge diagnoses, which were not independently confirmed. However, the estimated positive predictive value of a pneumonia diagnosis in the Danish National Registry of Patients is $90 \%$ (95\% CI $82 \%-95 \%)$ [3].
Another concern is that physicians may be more likely to hospitalise patients with infection if they are known to have high alcohol intake. Such bias would cause overestimation of the relative risks associated with high alcohol intake. However, a generally increased surveillance could probably not explain the observed differences in risk estimates between males and females. Conversely, persons with high alcohol intake and potentially associated socioeconomic or psychological problems may be less likely to seek medical advice and treatment [25], which would cause a bias in the opposite direction. We can only speculate about whether such detection bias may have been disproportionally strong among alcohol-drinking females versus males in our study. Finally, despite the relatively large size of our study, we had a limited ability to evaluate the risk of pneumonia-related hospitalisation for different combinations of alcohol amount and drinking frequency.

In the context of previous reports, our finding of a sex difference in the relationship between alcohol intake and pneumonia risk

TABLE 3 Hospitalisation rates for pneumonia among females according to alcohol intake, Denmark 1993-2008

\begin{tabular}{|c|c|c|c|c|c|}
\hline & \multicolumn{5}{|c|}{ Drinks $\cdot$ week $^{-1}$} \\
\hline & 0 & $1-6$ & $7-20$ & $21-35$ & $>35$ \\
\hline Females $\mathrm{n}$ & 644 & 12686 & 8854 & 2111 & 387 \\
\hline Pneumonia diagnosis $n$ & 35 & 472 & 329 & 98 & 10 \\
\hline $\begin{array}{l}\text { Incidence rate per } \\
1000 \text { person-yrs }\end{array}$ & 4.72 & 3.18 & 3.19 & 4.03 & 2.25 \\
\hline Adjusted HR $(95 \% \mathrm{Cl})^{\pi}$ & $1.26(0.89-1.79) p=0.19$ & 1.0 (ref.) & $1.01(0.88-1.17) p=0.87$ & $1.10(0.88-1.37) p=0.41$ & $0.54(0.29-1.01) p=0.06$ \\
\hline Adjusted HR $(95 \% \mathrm{Cl})^{+}$ & $1.17(0.82-1.68) p=0.38$ & 1.0 (ref.) & $1.03(0.89-1.19) p=0.70$ & $1.08(0.86-1.36) p=0.50$ & $0.48(0.25-0.93) p=0.03$ \\
\hline
\end{tabular}

HR: hazard ratio; Cl: confidence interval; ref.: reference. " ${ }^{*}$ : calculated using Cox's regression, with age as the underlying time variable; ": based on the crude model with additional adjustment for smoking status and body mass index; ${ }^{+}$: based on the crude model with additional adjustment for smoking status, body mass index and major chronic diseases. 


\begin{tabular}{|c|c|c|c|c|c|}
\hline & \multicolumn{5}{|c|}{ Frequency of alcohol consumption days $\cdot$ week $^{-1}$} \\
\hline & Never & $\leqslant 1$ & $2-4$ & $5-6$ & Daily \\
\hline \multicolumn{6}{|l|}{ Males } \\
\hline Incidence rate per 1000 person-yrs & 7.02 & 4.81 & 3.49 & 3.53 & 5.22 \\
\hline Crude HR $(95 \% \mathrm{Cl})^{\#}$ & $1.48(0.99-2.21) p=0.06$ & 1.0 (ref.) & $0.77(0.65-0.91) p=0.002$ & $0.78(0.64-0.96) p=0.02$ & $1.09(0.92-1.29) p=0.31$ \\
\hline Adjusted HR $(95 \% \mathrm{Cl})^{\circ}$ & $1.41(0.94-2.10) p=0.10$ & 1.0 (ref.) & $0.82(0.69-0.97) p=0.02$ & $0.82(0.67-1.01) p=0.06$ & $1.05(0.88-1.24) p=0.59$ \\
\hline Adjusted HR $(95 \% \mathrm{Cl})^{+}$ & $1.35(0.89-2.05) p=0.16$ & 1.0 (ref.) & $0.88(0.74-1.06) p=0.17$ & $0.88(0.71-1.08) p=0.23$ & $1.06(0.89-1.27) p=0.51$ \\
\hline \multicolumn{6}{|l|}{ Females } \\
\hline Adjusted HR $(95 \% \mathrm{Cl})^{\circ}$ & $1.14(0.80-1.61) p=0.48$ & 1.0 (ref.) & $0.81(0.70-0.95) p=0.01$ & $0.79(0.63-0.99) p=0.04$ & $0.90(0.75-1.09) p=0.29$ \\
\hline Adjusted HR $(95 \% \mathrm{Cl})^{+}$ & $1.06(0.74-1.52) \mathrm{p}=0.76$ & 1.0 (ref.) & $0.82(0.70-0.97) p=0.02$ & $0.78(0.62-0.99) \mathrm{p}=0.04$ & $0.90(0.74-1.09) p=0.28$ \\
\hline
\end{tabular}

HR: hazard ratio; $\mathrm{Cl}$ : confidence interval; ref.: reference. ${ }^{*}$ : calculated using Cox's regression, with age as the underlying time variable; ${ }^{\prime}$ : based on the crude model with additional adjustment for smoking status and body mass index; ${ }^{+}$: based on the crude model with additional adjustment for smoking status, body mass index and major chronic diseases.

accords with the results of a Spanish case-control study [16]. Based on 1,336 pneumonia cases and 1,336 age-, sex- and primary health centre-matched controls, Almirall et al. [16] found in crude analyses that an increasing amount of alcohol intake was associated with increasing risk of pneumonia in males but not in females. Unlike our study, however, the Spanish study did not examine whether smoking influenced results. Among US males from the Health Professionals' Follow-up Study and females from the Nurses' Health Study II, BAIK et al. [15] found no association between low or moderate alcohol intake and risk of pneumonia either in males after 6 yrs of follow-up or in females after 2 yrs of follow-up. Because their study population had a much lower reported alcohol intake than our Danish population, BAIK et al. [15] had limited power to observe the effect of heavy drinking. In addition to sex-based differences in alcohol pharmacokinetics, the sex difference observed in our study could be the result of chance because relatively few females (1.6\%) had an average consumption $>35$ drinks. week $^{-1}$. Also, in Denmark,

TABLE 5 Hospitalisation with pneumonia according to drinking frequency and alcohol intake

\begin{tabular}{|c|c|c|c|c|c|}
\hline \multirow{2}{*}{$\begin{array}{l}\text { Alcohol intake } \\
\text { drinks } \cdot \text { week }^{-1}\end{array}$} & \multicolumn{5}{|c|}{ Frequency of drinking alcohol days week $^{-1}$} \\
\hline & Never & $\leqslant 1$ & $2-4$ & $5-6$ & Daily \\
\hline \multicolumn{6}{|l|}{ Males } \\
\hline 0 & $1.38(0.92-2.07) p=0.12$ & & & & \\
\hline $1-6$ & & 1.0 (ref.) & $0.78(0.60-1.01) p=0.06$ & $1.41(0.72-2.78) p=0.31$ & $0.61(0.16-2.34) p=0.47$ \\
\hline $35-50$ & & & $1.04(0.47-2.29) p=0.93$ & $1.10(0.70-1.72) p=0.68$ & $1.08(0.84-1.40) p=0.54$ \\
\hline$>50$ & & $2.57(0.31-21.55) p=0.38$ & $2.80(0.99-7.92) p=0.05$ & $0.98(0.36-2.66) p=0.98$ & $1.70(1.29-2.24) p<0.001$ \\
\hline \multicolumn{6}{|l|}{ Females } \\
\hline 0 & $1.16(0.82-1.66) p=0.40$ & & & & \\
\hline $1-6$ & & 1.0 (ref.) & $0.75(0.60-0.93) p=0.01$ & $0.84(0.43-1.64) p=0.61$ & $0.50(0.13-2.03) p=0.35$ \\
\hline $7-20$ & & $1.28(0.90-1.82) p=0.18$ & $0.87(0.72-1.06) p=0.18$ & $0.81(0.62-1.06) p=0.12$ & $1.02(0.81-1.29) p=0.86$ \\
\hline
\end{tabular}

Data are present as hazard ratio $(95 \% \mathrm{Cl})$. The hazard ratio was calculated using Cox's regression, with age as the underlying time variable and with adjustment for smoking status and body mass index. Ref. reference. 
females in the highest socioeconomic classes tend to drink considerably more alcohol than females in the lower socioeconomic classes, and these differences according to socioeconomic status are reportedly larger than among males [26]. Although we were able to adjust for length of schooling and subsequent education, lack of adjustment for socioeconomic status may thus have led to social confounding associated with high alcohol intake among females more than among males. The previously mentioned earlier studies did not explore the association between drinking patterns and pneumonia risk.

Biological mechanisms underlying higher pneumonia risk among males with high alcohol intake may include development of chronic diseases. Excess alcohol consumption can lead to liver disease, including liver cirrhosis, which is characterised by a defect in innate immunity and a high susceptibility to infections [27]. Overconsumption of alcohol also contributes to cancers of the breast, oral cavity, larynx, pharynx and oesophagus [13], which may increase the likelihood of pneumonia. Other medical conditions associated with alcoholism that also could increase susceptibility to pneumonia include cardiomyopathy, hypertension, and malnutrition with vitamin deficiency [13, 28]. Nevertheless, after we added chronic diseases to the regression model, the association between high alcohol intake and pneumonia risk among males weakened but remained. Thus, our data show that chronic diseases alone do not explain the association. Other mechanisms may include the detrimental effects of chronic alcohol exposure per se on systemic and local immune responses [10, 12].

As other studies have done previously, we determined drinking patterns by combining information on average quantity with usual drinking frequency $[18,29]$. Our findings suggest that infrequent heavy drinking is particularly harmful concerning pneumonia risk. For the same amount of alcohol intake, an infrequent drinking pattern compared to a frequent drinking pattern would lead to a higher alcohol concentration in the gastrointestinal tract and in the blood which, in turn, could lead to an escalation of the harmful effects of alcohol [18]. It is plausible that infrequent excessive alcohol consumption leads to episodes of decreased consciousness, vomiting, immobility and body cooling. Our study's long-term follow-up design prevented examination of the effects of acute alcohol exposure on pneumonia risk.
Among males, moderate alcohol consumption tended to have a small, statistically nonsignificant protective effect against pneumonia, compared with drinking less. It has been suggested that moderate consumption of alcohol may enhance immune function through a positive effect on lymphocyte count and on pro-inflammatory cytokine cascades [30]. Components such as polyphenols, antioxidants and vitamins that are present in beer or wine may also have beneficial effects on immunity [30]. TAKKOUCHE et al. [31] found that consumption of wine, in particular red wine, was inversely associated with the risk of common cold among Spanish university faculty staff, with an adjusted incidence rate ratio for drinkers of $>14$ glasses of wine per week of 0.6 (95\% CI 0.4-0.8) compared with nondrinkers. Similarly, among 391 persons intentionally exposed to respiratory virus and 26 persons given saline, COHEN et al. [32] showed that moderate consumption of alcohol was associated with decreased risk of developing clinical colds.

An additional finding was our observation that male alcohol abstainers had a higher pneumonia risk than males who drank 1-6 drinks. week $^{-1}$. However, there are few total abstainers in the Danish Diet, Cancer and Health Study, and many of these have reported a previously high alcohol intake. Similarly, a British study showed that middle-aged, male nondrinkers are likely to be ex-drinkers, older and have higher prevalence of a wide range of diseases and drug treatments compared to light or moderate drinkers [33].

In conclusion, our data show that consumption of $>50$ alcoholic drinks per week among males and infrequent heavy drinking are positively associated with risk of pneumonia-related hospitalisation. In contrast, moderate alcohol intake had no detrimental effect on pneumonia risk.

\section{APPENDIX}

International Classification of Diseases (ICD) codes for major chronic diseases are shown in table 6 . The codes were used both to exclude people with major chronic diseases at baseline and to control for chronic diseases diagnosed during follow-up.

\section{SUPPORT STATEMENT}

This work was supported by Klinisk Epidemiologisk Forskningsfond at Aarhus University (Aarhus, Denmark) and the Danish Cancer Society (Copenhagen, Denmark).

TABLE 6 International Classification of Diseases (ICD) codes for major chronic diseases

$\begin{array}{lll}\text { Disease category } & \text { ICD-8 } & \text { ICD-10 }\end{array}$

Diseases related directly to alcoholism

Cancer

Cardiovascular diseases

Chronic pulmonary diseases

Other
$571.09,571.10 ; 577.10$

$140-207 ; 275.59$

400-404; 410; 427.09-427.11; 427.19; 428.99; 430-438;

$440-445 ; 782.49$

490-493; 515-518

070.00; 070.02; 070.04; 070.06; 070.08; 079.83; 135.99; 249; 250; 290.09-290.19; 293.09; 344; 446; 456.0; 571.11 571.19; 571.99; 530.91; 530.98; 530.99; 531-534; 573.00; 573.01; 573.04; 580-584; 590.09; 593.19; 712; 716; 734 ;

$753.1 ; 792$
G31.2; G62.1; G72.1; 142.6; K29.2; K70; K86.0 C00-C85; C88; C90-C96

G45; G46; I10-I15; I21-I23; 150; 160-169; 170-174; 177

J40-J47; J60-J67; J68.4; J70.1; J70.3; J84.1; J92.0

J96.1; J98.2; J98.3

B20-B24; B15.0; B16.0; B16.2; B18; B19.0; D86; E10-E14; F00-F03; F05.1; G30; G81; G82; H36.0; I85; K21; K22.1; K25-K28; K71-K73; K74; K76.0; K76.6; M05; M06; M08; M09; M30-M36; N00-N05; N07; N11; N14; N17-N19; O24.0-O24.3; O24.5-O24.9; Q61 


\section{STATEMENT OF INTEREST}

None declared.

\section{REFERENCES}

1 Ruhnke GW, Coca-Perraillon M, Kitch BT, et al. marked reduction in 30-day mortality among elderly patients with communityacquired pneumonia. Am J Med 2011; 124: 171-178.

2 Fry AM, Shay DK, Holman RC, et al. Trends in hospitalizations for pneumonia among persons aged 65 years or older in the United States, 1988-2002. JAMA 2005; 294: 2712-2719.

3 Thomsen RW, Riis A, Nørgaard M, et al. Rising incidence and persistently high mortality of hospitalised pneumonia: a 10-year population-based study in Denmark. J Intern Med 2006; 259: 410-417.

4 Trotter CL, Stuart JM, George R, et al. Increasing hospital admissions for pneumonia, England. Emerg Infect Dis 2008; 14: 727-733.

5 van Gageldonk-Lafeber AB, Bogaerts MA, Verheij RA, et al. Time trends in primary-care morbidity, hospitalization and mortality due to pneumonia. Epidemiol Infect 2009; 137: 1472-1478.

6 de Roux A, Cavalcanti M, Marcos MA, et al. Impact of alcohol abuse in the etiology and severity of community-acquired pneumonia. Chest 2006; 129: 1219-1225.

7 Fernandez-Sola J, Junque A, Estruch R, et al. High alcohol intake as a risk and prognostic factor for community-acquired pneumonia. Arch Intern Med 1995; 155: 1649-1654.

8 Koivula I, Sten M, Makela PH. Risk factors for pneumonia in the elderly. Am J Med 1994; 96: 313-320.

9 Ruiz M, Ewig S, Torres A, et al. Severe community-acquired pneumonia. Risk factors and follow-up epidemiology. Am J Respir Crit Care Med 1999; 160: 923-929.

10 Samokhvalov AV, Irving HM, Rehm J. Alcohol consumption as a risk factor for pneumonia: a systematic review and meta-analysis. Epidemiol Infect 2010; 138: 1789-1795.

11 World Health Organization, Dept of Mental Health and Substance Abuse. Global Status Report on Alcohol 2004. Geneva, World Health Organization, 2004. www.who.int/substance_abuse/ publications/global_status_report_2004_overview.pdf.

12 Moss M. Epidemiology of sepsis: race, sex, and chronic alcohol abuse. Clin Infect Dis 2005; 41: Suppl. 7, S490-S497.

13 Rehm J, Baliunas D, Borges GL, et al. The relation between different dimensions of alcohol consumption and burden of disease: an overview. Addiction 2010; 105: 817-843.

14 Almirall J, Bolibar I, Balanzó X, et al. Risk factors for communityacquired pneumonia in adults: a population-based case-control study. Eur Respir J 1999; 13: 349-355.

15 Baik I, Curhan GC, Rimm EB, et al. A prospective study of age and lifestyle factors in relation to community-acquired pneumonia in US men and females. Arch Intern Med 2000; 160: 3082-3088.

16 Almirall J, Bolibar I, Serra-Prat $M$, et al. New evidence of risk factors for community-acquired pneumonia: a population-based study. Eur Respir J 2008; 31: 1274-1284.
17 Tolstrup J, Jensen MK, Tjønneland A, et al. Prospective study of alcohol drinking patterns and coronary heart disease in females and men. BMJ 2006; 332: 1244-1248.

18 Tolstrup JS, Jensen MK, Tjønneland A, et al. Drinking pattern and mortality in middle-aged men and females. Addiction 2004; 99: 323-330.

19 Mumenthaler MS, Taylor JL, O'Hara R, et al. Gender differences in moderate drinking effects. Alcohol Res Health 1999; 23: 55-64.

20 Kornum JB, Nørgaard M, Dethlefsen C, et al. Obesity and risk of subsequent hospitalisation with pneumonia. Eur Respir J 2010; 36: 1330-1336.

21 Tjønneland A, Olsen A, Boll K, et al. Study design, exposure variables, and socioeconomic determinants of participation in Diet, Cancer and Health: a population-based prospective cohort study of 57,053 men and females in Denmark. Scand J Public Health 2007; 35: 432-441.

22 Overvad K, Tjønneland A, Haraldsdottir J, et al. Development of a semiquantitative food frequency questionnaire to assess food, energy and nutrient intake in Denmark. Int J Epidemiol 1991; 20: 900-905.

23 Tjønneland A, Overvad K, Haraldsdottir J, et al. Validation of a semiquantitative food frequency questionnaire developed in Denmark. Int J Epidemiol 1991; 20: 906-912.

24 Charlson ME, Pompei $\mathrm{P}$, Ales $\mathrm{KL}$, et al. A new method of classifying prognostic comorbidity in longitudinal studies: development and validation. J Chronic Dis 1987; 40: 373-383.

25 Arendt JN, Jensen BT, Nexoe J, et al. Lavindkomst og antal kontakter med almen praksis [Low income and number of contacts with general practice]. Ugeskr Laeger 2010; 172: 1591-1597.

26 Ekholm O, Kjøller M, Davidsen M, et al., eds. Sundhed og sygelighed i Danmark \& udviklingen siden 1987 [Health and sickness in Denmark and development since 1987]. Copenhagen Statens Institut for Folkesundhed, 2006.

27 Leber B, Mayrhauser U, Rybczynski M. Innate immune dysfunction in acute and chronic liver disease. Wien Klin Wochenschr 2009; 121: 732-744.

28 Manari AP, Preedy VR, Peters TJ. Nutritional intake of hazardous drinkers and dependent alcoholics in the UK. Addict Biol 2003; 8: 201-210.

29 Mukamal KJ, Conigrave KM, Mittleman MA, et al. Roles of drinking pattern and type of alcohol consumed in coronary heart disease in men. N Engl J Med 2003; 348: 109-118.

30 Romeo J, Wärnberg J, Nova E, et al. Moderate alcohol consumption and the immune system: a review. Br J Nutr 2007; 98: Suppl. 1, S111-S115.

31 Takkouche B, Regueira-Méndez C, García-Closas R, et al. Intake of wine, beer, and spirits and the risk of clinical common cold. Am J Epidemiol 2002; 155: 853-858.

32 Cohen S, Tyrrell DA, Russel MA, et al. Smoking, alcohol consumption, and susceptibility to the common cold. Am J Public Health 1993; 83: 1277-1283.

33 Wannamethee G, Shaper AG. Men who do not drink: a report from the British Regional Heart Study. Int J Epidemiol 1988; 17: $307-316$ 